\title{
Automedicación y factores relacionados en trabajadores de Salud que laboran en el Instituto Materno Perinatal de Lima 2011
}

\author{
Elsy Mini, Julio Medina, Luis Rojas, Joece Butrón, Verónica Peralta, Renzo Aranda, \\ María Lévano, Rocío Varas, Yuliana Vicuña, Ericson Gutiérrez \\ Instituto Nacional Materno Perinatal, Lima, Perú
}

Objetivos: Determinar la prevalencia de automedicación y sus principales características en trabajadores de Salud que laboran en el Instituto Nacional Materno Perinatal de Lima.

Diseño: Estudio descriptivo transversal realizado

Institución: Instituto Nacional Materno Perinatal, Lima, Perú.

Participantes: Trabajadores de Salud, asistenciales y administrativos; no se incluyó médicos, enfermeras, obstetrices, odontólogos ni químicos farmacéuticos.

Intervenciones: Entre julio y setiembre de 2011 se realizó entrevista a 226 trabajadores aplicando un cuestionario que fue validado mediante juicio de expertos y una prueba piloto; tipo de muestreo aleatorio simple; los cálculos fueron realizados con nivel de confianza de $95 \%$.

Principales medidas de resultados: Automedicación.

Resultados: La prevalencia de automedicación fue $79 \%$. El $63 \%$ refirió su uso por falta de tiempo para ir al médico y su uso favorable como antecedente. El $95 \%$ conocía que el uso de la automedicación es un riesgo para su salud. El 91\% lo adquirió en farmacias y boticas, donde $92 \%$ refiere que no le solicitaron receta médica.

Conclusiones: Existió una elevada prevalencia de automedicación en estos trabajadores, a pesar de encontrase en una institución de salud. La falta de tiempo, el antecedente de uso favorable y la fácil adquisición de medicamentos fueron las características más importantes de la automedicación.

Palabras clave: Trabajadores de Salud, automedicación.

\section{Conocimiento sobre interculturalidad en salud, de los alumnos de la EAP de Medicina de la UNMSM}

\author{
Alicia Fernández, Zully Acosta, Enma Cambillo \\ Instituto de Investigaciones Clinicas, Facultad de Medicina, UNMSM, y Hospital Dos de Mayo, Lima, Perú
}

Objetivos: Determinar el grado de conocimiento en interculturalidad en Salud, en alumnos de la EAP de Medicina de la Facultad de Medicina, UNMSM.

Diseño: Descriptivo, transversal.

Institución: Instituto de Investigaciones Clínicas, Facultad de Medicina, UNMSM, y Hospital Dos de Mayo, Lima, Perú.

Participantes: Internos de medicina de la UNMSM.

Intervenciones: Encuesta validada a 101 internos de Medicina, 66 alumnos de sexo masculino y 35 de sexo femenino, con edades comprendidas entre 22 y 34 años, donde se evaluó 8 variables.

Principales medidas de resultados: Porcentaje de grado de conocimiento sobre interculturalidad en Salud.

Resultados: Los alumnos definieron correctamente, en su mayoría $(79,2 \%)$, el término de interculturalidad en Salud; no habian tenido contacto laboral con las poblaciones indígenas ni tampoco hablan quechua o aymara. En la capacitación de recursos humanos, 95\% de los encuestados opinó que los cursos clínicos deben incorporar aspectos de la situación de salud de esta población, elaborar tesis y trabajos de investigación sobre el tema y compilar información sobre los recursos terapéuticos de la medicina tradicional.

Conclusiones: Los recursos humanos de la Escuela de Medicina deben ser fortalecidos en sus capacidades y competencias a través de la incorporación de la interculturalidad en salud como eje horizontal, en los cursos de grado de Medicina, Pediatría, Gineco-Obstetricia y Salud Pública.

Palabras clave: Interculturalidad en salud, pueblos indígenas. 